\title{
LOS Zoophycos DEL BAJOCIENSE-BATHONIENSE DE LA PRAIA DA MARETA (ALGARVE, PORTUGAL): ARQUITECTURA Y FINALIDADES EN RÉGIMEN DE DOMINANCIA ECOLÓGICA
}

Trabajo presentado a las XVIII Jornadas de Paleontología y II Congreso Ibérico de Paleontología Universidad de Salamanca

Salamanca, 24-29 de Septiembre de 2002

\author{
Carlos NETO DE CARVALHO ${ }^{1,2}$ y Nuno P.C. \\ RODRIGUES $S^{2,3}$
}

\begin{abstract}
${ }^{1}$ Centro de Geologia da Universidade de Lisboa, Bloco C2, $5^{\circ}$ piso, Campo Grande, P-1700 Lisboa, Portugal. E-mail: praedichnia@hotmail.com.

${ }^{2}$ Grupo PALEO - Grupo de Paleontologia do Museu Nacional de História Natural. Rua da Escola Politécnica, n ${ }^{\circ}$, P-1294 Lisboa Codex, Portugal. ${ }^{3}$ Departamento de Paleontología e Instituto de Geología Económica (C.S.I.C.U.C.M.), Facultad de Ciencias Geológicas, Universidad Complutense de Madrid, Ciudad Universitaria, 28040 Madrid, España. E-mail: nuno.carla@teleline.es.
\end{abstract}

Neto de Carvalho, C. y Rodrigues, N. P. C. 2003. Los Zoophycos del Bajociense-Bathoniense de la Praia da Mareta (Algarve, Portugal): Arquitectura y finalidades en régimen de dominancia ecológica. [The Zoophycos From the Bajocian-Bathonian of Praia da Mareta (Algarve, Portugal): Archictecture and purposes in ecological dominance regime.] Revista Española de Paleontología, 18 (2), 229-241. ISSN 0213-6937.

\begin{abstract}
Zoophycos is known in Portugal since the biostratigraphical works of Paul Choffat, published in 1880, also by the junior synonyms Cancellophycus, Taonurus and Alectorurus. This ichnogenus occurs in the portuguese stratigraphical record from the late Arenig to the present, being particularly common in the Toarcian-to-Callovian forereefal calciturbiditic sequences from the Lusitanian and Algarve basins. Historically, the portuguese Zoophycos were identified as algae, Problematica, algae tallus, algae or coral disturbance imprints and, just more recently, as product of animal palaeobiological activity.

This study is based on the Zoophycos ichnocoenoses found in omission surfaces distributed along the "Margas e calcários detríticos com Zoophycos da Praia da Mareta" formation stratotype, from the upper Bajocian-lower Bathonian. The constructional model for Praia da Mareta Zoophycos can be explained as an helicoid multi-spreite with logarithmic spirals, formed by U-shaped Rhizocorallium-like lamina, developed oblique and centripetally starting from a coiling axis. The spreite lamella are dominated by a micritic filling, faecal pellets devoided, very unlike the surrounding non-bioturbated sediment, which indicates mining strips activity by an endodepositivorous vermiform producer.

The Zoophycos-Chondrites ichnoguild, as distal expression of the Glossifungites ichnofacies, dominates the forereefal/distal homoclinal ramp facies of the Lusitanian and Algarve basins. In the Praia da Mareta sequence, the Zoophycos producers appears in large densities, certainly associated to ecological maxima, without biotic or environmental evidences of opportunistic ichnocoenoses. The Zoophycos climax communities generally tend to possess ecophenotipic antisymmetry at coiling level. Particularly in the studied samples, the proximity to the tolerance limits by the Zoophycos producers, caused by the anoxia increase, nutritional deplection and/or ecospace restriction by multigenerational overpopulation, could be responsible for the hormetical development of directional asymmetry, without adaptive significance.

The combination of the new data for Zoophycos in this work, in a national or even global stratigraphical context, can show the ecological conditions which allowed the evolution of the Zoophycos ichnofacies. The timeenvironment diagrams for Zoophycos elaborated with the portuguese data and with more than one hundred bibliographical references from all over the world, permit us to verifiy that in Portugal exists the same worldwhile ecological radiation tendency to deep-sea colonization of this complex feeding behaviour. However, these new data differ from the previously one, obtained by others, specially in the deep-sea first appearance and definite restriction to slope and basinal enviroment timings. In fact, the Zoophycos producers were pioneer settlers, altough accidental, of the basinal bottoms since the Lower Ordovician, refering to slope-abissal niches exclusively and typifying the Zoophycos ichnofacies only after the Cretaceous-Tertiary extinction event.
\end{abstract}

Keywords: Composite Zoophycos, Bajocian-Bathonian, behaviour, Glossifungites ichnofacies, direccional asymmetry, hormesis, ecological radiation. 


\section{RESUMEN}

El icnogénero Zoophycos es conocido en Portugal desde los trabajos de Paul Choffat, publicados en 1880. Presenta una distribución estratigráfica que se extiende, en el territorio portugués, desde el Arenig superior hasta la actualidad, siendo muy frecuente en las secuencias calciturbidíticas del Toarciense al Calloviense de las cuencas Lusitánica y del Algarve.

El presente estudio se centra en las icnocenosis de Zoophycos que se encuentran en superficies de omisión a lo largo del estratotipo de la unidad "Margas e calcários detríticos com Zoophycos da Praia da Mareta", de edad Bajociense superior-Bathoniense inferior. La forma de los Zoophycos compuestos de la Praia da Mareta se puede explicar por un multi-spreite helicoidal con espiral logarítmica, constituido por láminas de galerías en U, del tipo Rhizocorallium, desarrolladas oblicuamente y de modo centrípeto a partir de un eje de enrollamiento. Las lamelas están constituidas por sedimento micrítico, muy pobre en componentes, lo que indica una actividad en bandas de prospección por un productor vermiforme detritívoro.

El icnogremio de Zoophycos-Chondrites domina las facies de frente arrecifal/rampa homoclinal externa de las cuencas Lusitánica y del Algarve. En los niveles estudiados, los productores de Zoophycos aparecen en grandes densidades y no muestran características bióticas o ambientales de icnocenosis oportunistas. Las comunidades clímax de Zoophycos tienden a poseer antisimetría ecofenotípica del enrollamiento. En los ejemplos de la Praia da Mareta, la aproximación de los productores de Zoophycos a los límites de tolerancia, ocasionado por incremento progresivo de la anoxia, depleción nutricional y/o restricción del ecoespacio por sobrepoblación, habría llevado al desarrollo hormético de asimetría direccional, careciendo de significado adaptativo.

Integrando los datos obtenidos en este trabajo para Zoophycos, en un contexto estratigráfico nacional o incluso global, se pueden visualizar los factores ecológicos que han permitido la evolución temporal de la icnofacies de Zoophycos. La elaboración de diagramas cronológico-ambientales a partir de los datos conocidos sobre los Zoophycos de Portugal y de más de un centenar de referencias bibliográficas de todo el mundo, ha permitido verificar que en Portugal existe la misma tendencia de radiación ecológica hacia una colonización de áreas profundas, difiriendo en algunos aspectos de los datos obtenidos anteriormente por otros autores. De hecho, los Zoophycos son colonizadores pioneros, pero fortuitos, de los fondos marinos (Ordovícico Inferior), restringiéndose exclusivamente a los nichos batiales-abisales después de la gran extinción de finales del Cretácico.

Palabras clave: Zoophycos compuestos, Bajociense-Bathoniense, comportamiento, icnofacies de Glossifungites, asimetría direccional, hormesis, radiación ecológica.

\section{LOCALIZACIÓN GEOGRÁFICA Y DISTRIBUCIÓN ESTRATIGRÁFICA EN PORTUGAL}

El icnogénero Zoophycos Massalongo, 1855 es conocido en Portugal desde los trabajos estratigráficos de Paul Choffat en las cuencas mesozoicas portuguesas, apareciendo en la bibliografía también bajo las designaciones sinónimas de Cancellophycus De Saporta, 1872 (vide Ruget-Perrot, 1961; Rocha, 1971; Teixeira, 1978), C. scoparius De Saporta, 1872 (vide Choffat, 1880), Taonurus procerus Heer, 1877 (vide Heer, 1881; Choffat, 1887, 1894), T. scoparius Thiolliére, 1858 (vide Heer, 1881) y Alectorurus cincinnaticus Schimper, 1869 (vide Delgado, 1908). Presenta una distribución estratigráfica que se extiende, en el territorio portugués, desde el Arenig superior (Piçarra, 2000; Romano, com. pers.) hasta la actualidad (Wetzel y Werner, 1981), siendo muy frecuente en las secuencias calciturbidíticas, de margocalizas, del Toarciense al Calloviense de las cuencas Lusitánica y del Algarve. La figura 1 muestra la distribución geográfica de Zoophycos en Portugal, indicándose las unidades estratigráficas don- de aparecen y la edad de cada afloramiento (las cuencas terciarias no se han representado por no haber evidencias de Zoophycos en sus sedimentos). Este mapa fue realizado con una base bibliográfica (publicaciones) y datos inéditos, constituyendo, en su conjunto, una síntesis de los afloramientos de Portugal que contienen estos icnofósiles, destacándose sobre todo el yacimiento estudiado en el presente trabajo. Se indica también la localización de los niveles estratigráficos con icnocenosis que han sido objeto de comparación en el análisis paleoecológico de Zoophycos.

Aunque se estén construyendo, en este preciso momento, estructuras de este tipo en la rampa continental paralela a la línea de costa portuguesa, su productor vermiforme continúa siendo, hoy en día, una de las mayores incógnitas de la Icnología. Siguiendo la evolución histórica del conocimiento paleoicnológico global, los Zoophycos identificados en Portugal han sido descritos como algas, Problematica (Heer, 1881), talos de algas (Choffat, 1887), marcas de agitación de algas o de coralarios (Teixeira, 1978) y, sólo más recientemente, como producto de la actividad paleobiológica de animales (Rocha, 1971). Sin embargo, son raros los trabajos que abordan estos icnofósiles en Portugal y normalmente lo hacen con un carácter esencialmente 


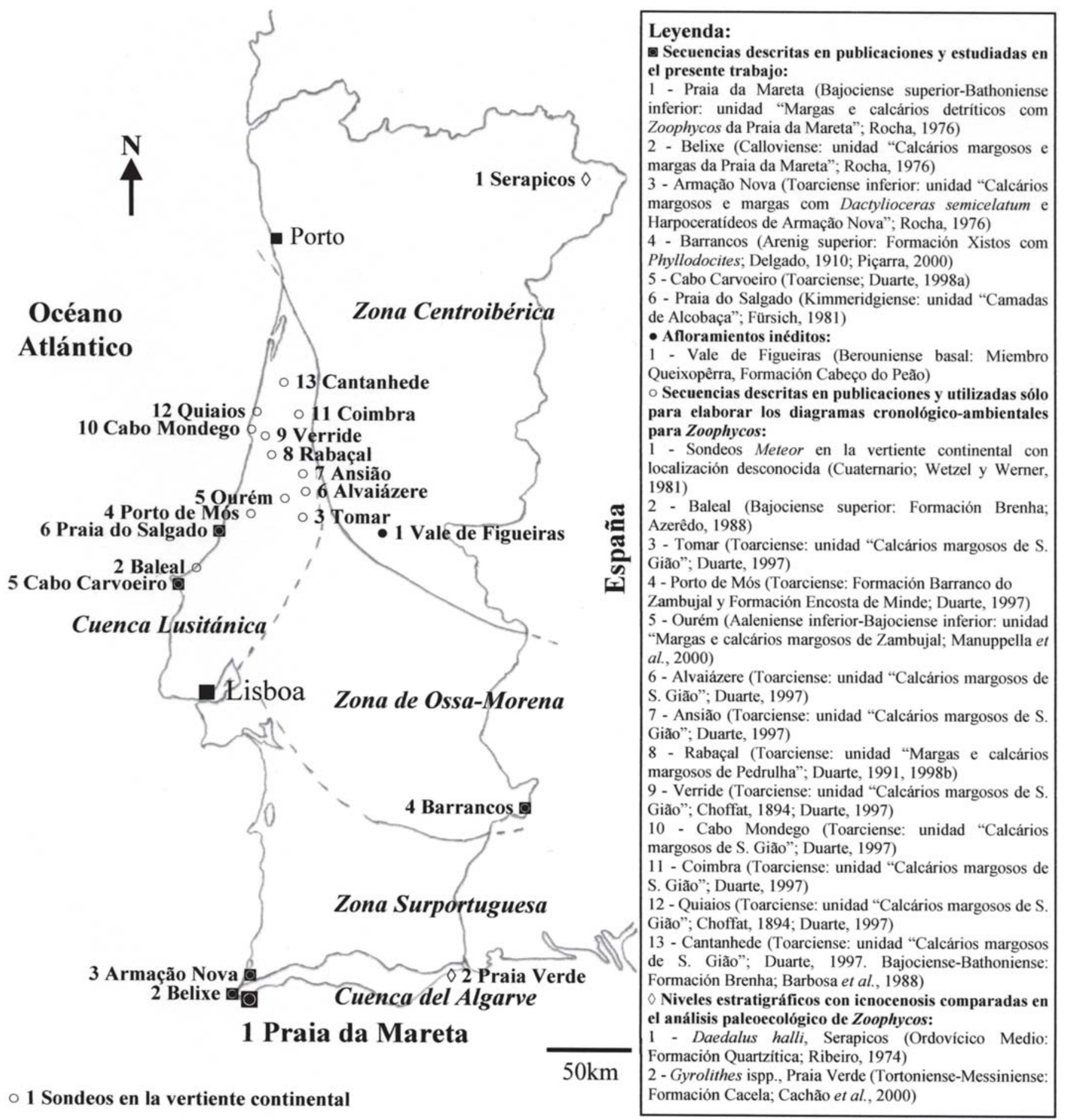

Figura 1. Distribución geográfica y estratigráfica de Zoophycos en Portugal, con referencias a los locales estudiados y a las publicaciones consultadas.

Geographical and stratigraphical distribution of Zoophycos in Portugal, with references to the studied sites and consulted publications.

de interpretación paleoambiental (vide Azerêdo, 1988; Duarte, 1991, 1997, 1998a, 1998b).

El presente estudio de funcionalidad y ecología se centra en los magníficos ejemplos de icnocenosis de Zoophycos que se encuentran en superficies de omisión a lo largo de la unidad "Margas e calcários detríticos com Zoophycos da Praia da Ma- reta", que aflora en los acantilados de Sagres (Algarve). Esta secuencia, con $25 \mathrm{~m}$ de espesor, de edad Bajociense superiorBathoniense inferior (Rocha, 1976), está dominada por litofacies de calizas margosas (bio)clásticas en bancos decimétricos, con niveles margoarcillosos, a veces muy bioturbados, entre los que se registran otros niveles con olistostromas (Fig. 2). 


\section{Afloramiento B}

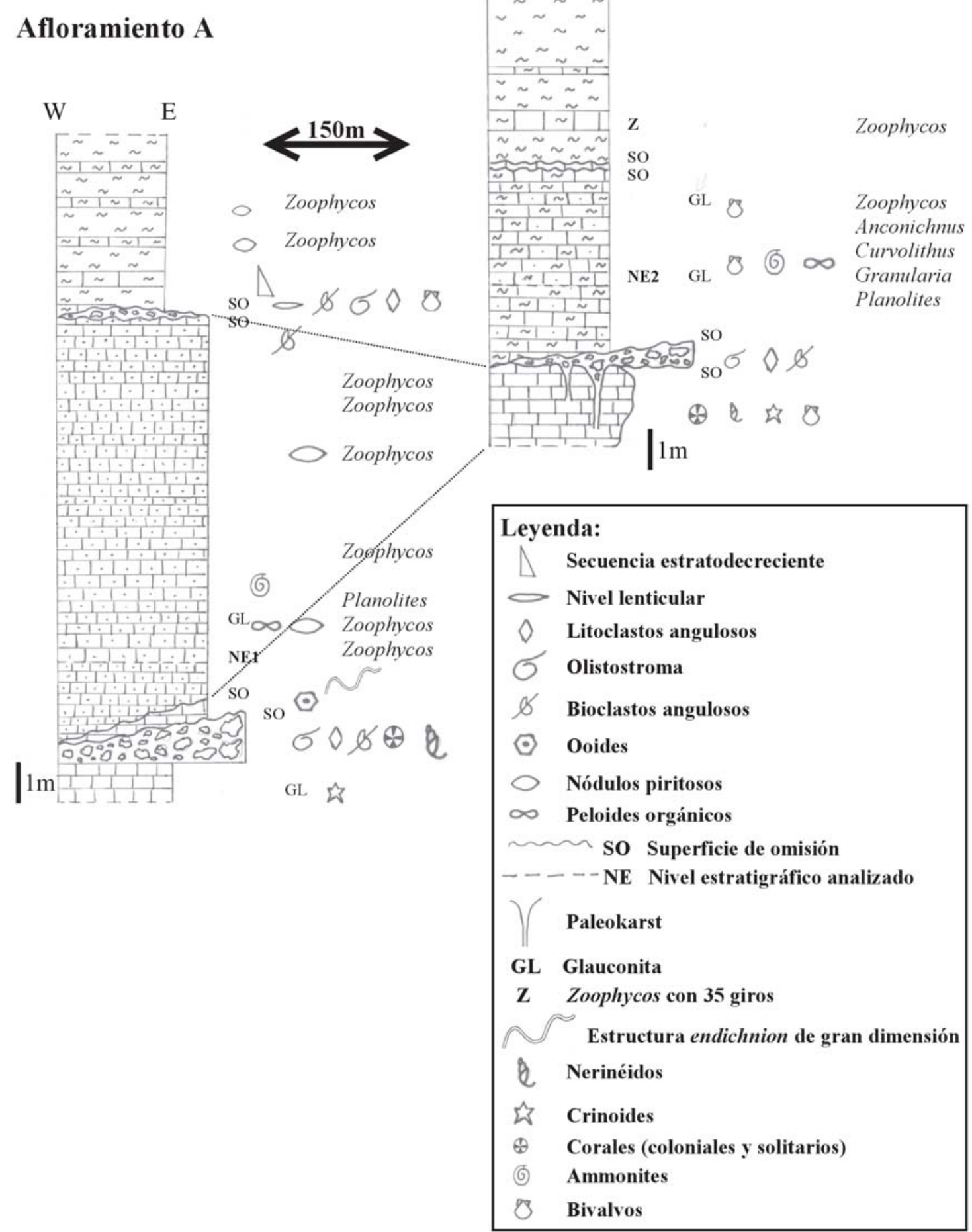

Figura 2. Sucesión litológica en el estratotipo de la unidad "Margas e calcários detríticos com Zoophycos da Praia da Mareta", con la situación de los niveles estratigráficos analizados (NE1 y NE2) y la distribución vertical de las estructuras sedimentarias observadas, fósiles e icnoasociaciones.

Lithological succession in the type section of the "Margas e calcários detríticos com Zoophycos da Praia da Mareta" formation, showing the location of the studied stratigraphical horizons (NE1 and NE2) and the vertical distribution of sedimentary structures, main fossil assemblages and ichnoassociations. 
La detallada observación de centenares de ejemplares de Zoophycos en la unidad estudiada, así como muchas otras observaciones llevadas a cabo en las secuencias mesozoicas portuguesas (Fig. 1), han permitido desarrollar un modelo explicativo de aplicación regional para las formas de comportamiento interpretadas en Zoophycos. Estos datos, en conjunto con los resultados obtenidos a partir del análisis bibliográfico realizado, han permitido contribuir para la comprensión de la paleoecología y evolución espacio-temporal de este icnogénero.

\section{LA ARQUITECTURA COMPUESTA DE Zoophycos: COMPLEJIDAD ESTRUCTURAL PARA LA OPTIMIZACIÓN TRIDIMENSIONAL DEL TRATAMIENTO DE LOS RECURSOS}

Las dos comunidades de Zoophycos estudiadas (NE1 y NE2) aparecen en niveles decimétricos de biopelmicrita
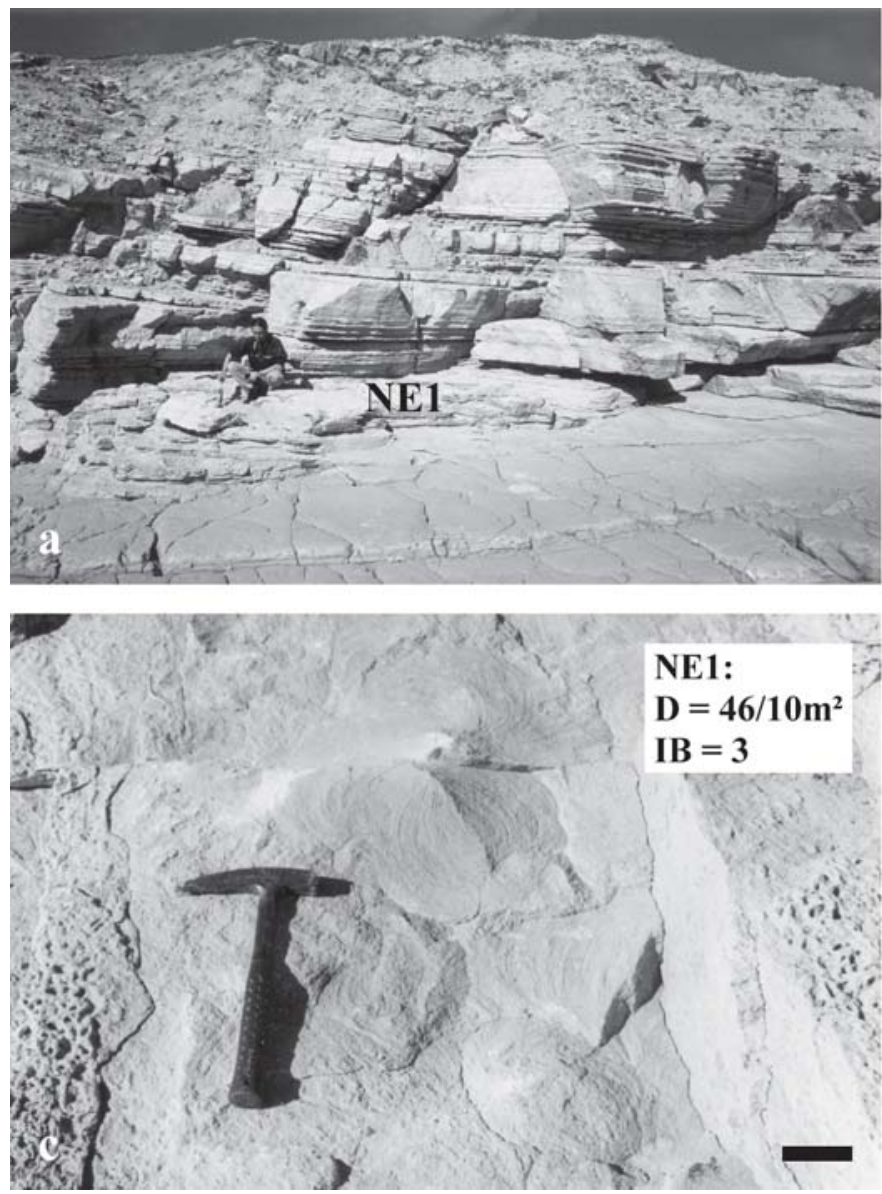

wackestone. NE1 presenta una densidad de 46 estructuras en $10 \mathrm{~m}^{2}$ y posee un índice de bioturbación 3 en el plano de estratificación (sensu Miller y Smail, 1997; fig. 3a y $3 \mathrm{c}$ ), que se puede considerar común a lo largo de la secuencia del Toarciense-Calloviense del Algarve occidental; para NE2 se ha contabilizado una densidad impresionante de 112 estructuras en $10 \mathrm{~m}^{2}$ y un índice de bioturbación 5 (Fig. 3b y 3d).

La forma de los Zoophycos compuestos (sensu Ekdale y Lewis, 1991; Ekdale, 1992) de la Praia da Mareta se puede explicar por un multi-spreite helicoidal con espiral logarítmica, constituido por galerías en U, del tipo Rhizocorallium (Fig. 4a), en relieve completo y protrusivas (Fig. 4b), desarrolladas oblicuamente y de modo centrípeto (Fig. 4c) a partir de una zona axial. Este eje de enrollamiento corresponde a las varias terminaciones de las galerías en U (Fig. 4d), dirigidas bruscamente hacia la interfase sedimento-agua. Su pequeña truncación erosiva por el plano de estratificación significa que los Zoophycos de la Praia da Mareta ocuparon un nivel relativamente superficial. Las galerías en U van aumentando
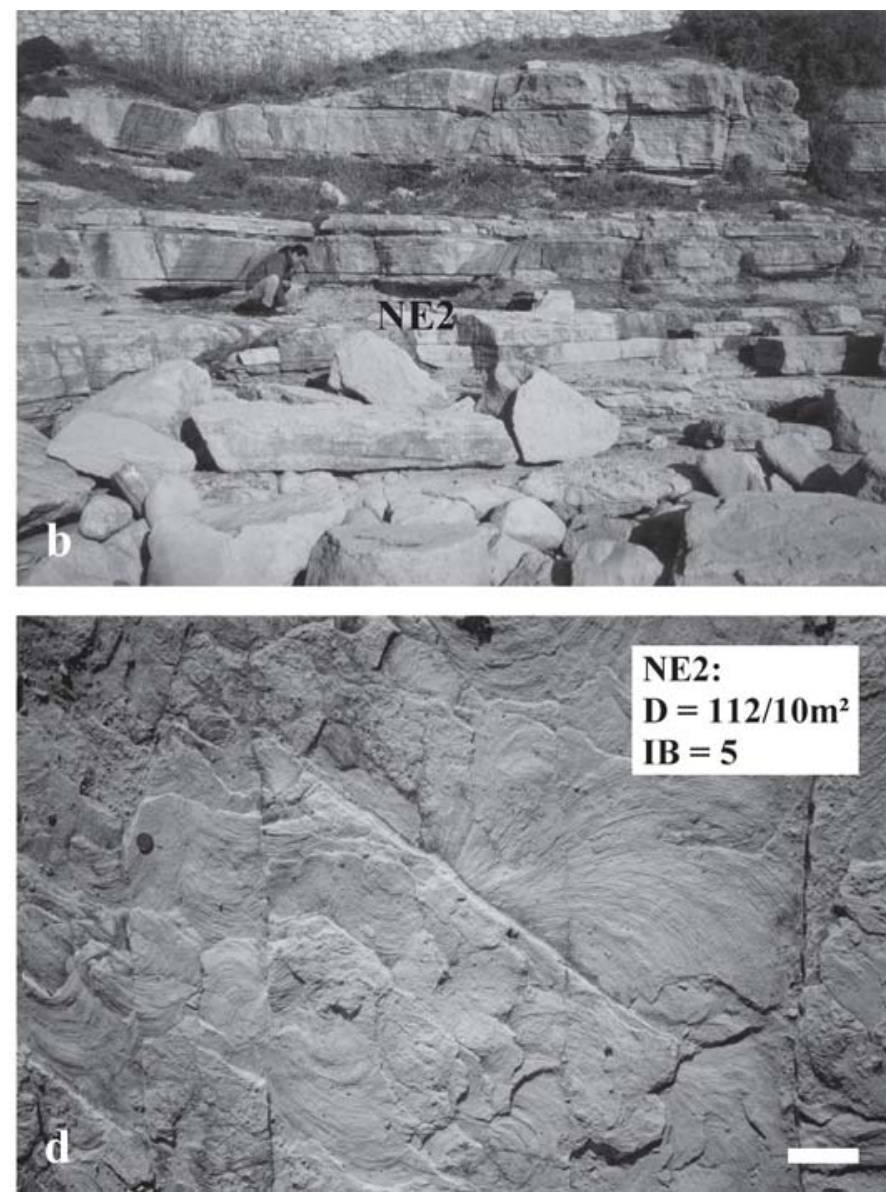

Figura 3. Situación de los niveles estratigráficos estudiados en el estratotipo de la unidad "Margas e calcários detríticos com Zoophycos da Praia da Mareta". a. NE1. b. NE2. c. Bioturbación por Zoophycos en NE1. d. Bioturbación por Zoophycos en NE2. $\mathrm{D}=$ densidad de Zoophycos; $\mathrm{IB}=$ índice de bioturbación. Escala $=10 \mathrm{~cm}$ (en $\mathrm{c}$ y d).

Location of the studied stratigraphical horizons in the type section of the "Margas e calcários detriticos com Zoophycos da Praia da Mareta" formation. a. NE1. b. NE2. c. Bioturbation by Zoophycos producers in NE1. d. Bioturbation by Zoophycos producers in NE2. $D=$ Zoophycos density; $I B=$ bioturbation index. Scale bar $=10 \mathrm{~cm}$ (in $\mathrm{c}$ and $d$ ). 

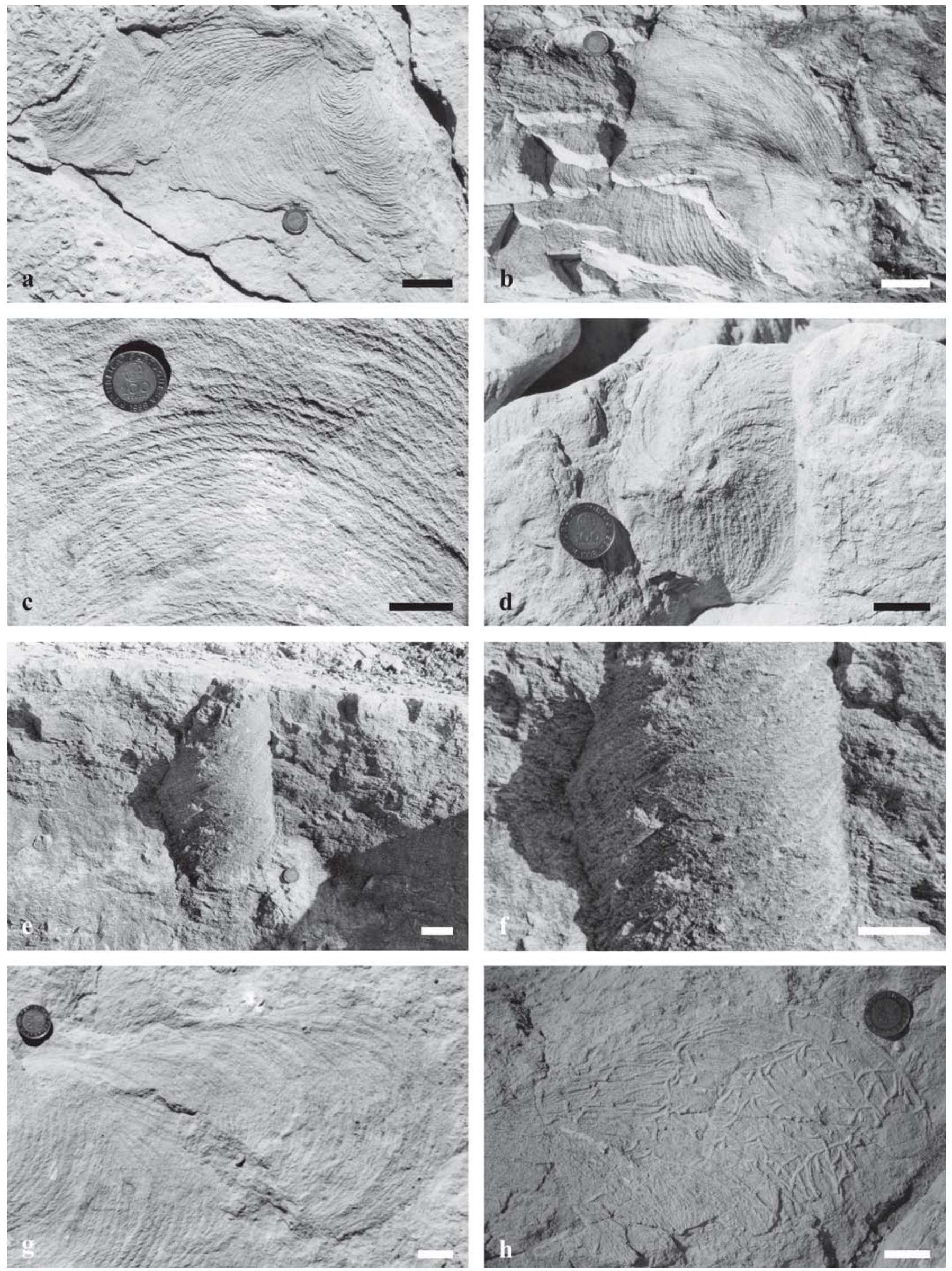
de área según se produce el desplazamiento perpendicular al eje de enrollamiento o se mantienen constantes a lo largo de vectores de expansión, sufriendo incrementos dimensionales en los sucesivos vectores y en profundidad (Fig. 4a). No se han observado galerías marginales. Las láminas (spreiten) pueden tener más de $40 \mathrm{~cm}$ de diámetro y se han contabilizado hasta 35 giros en la estructura helicoidal (posiblemente limitados espacialmente por la capacidad de circulación de agua con oxígeno; Ekdale, 1992; Figs. 4e y 4f). A veces, el margen de las láminas es lobulado (Fig. 4g), indicando una prospección en sustrato desocupado o seeking behaviour (Bromley, 1991). Las lamelas (Fig. 4c) están constituidas por sedimento micrítico, muy pobre en componentes clásticos, contrastando con una matriz rica en peloides orgánicos, lo que favorece una actividad en bandas de prospección (stripmining) por un productor vermiforme detritívoro durante un intervalo de tiempo en el cual no hubo crecimiento del organismo, teniendo en cuenta el diámetro constante de la galería en U. De este modo, el modelo de Zoophycos de la Praia da Mareta, análogamente a los Zoophycos observados en el Toarciense-Calloviense del Algarve occidental, no encaja en el modelo sanitario de Kotake (1989), que está caracterizado por un relleno fecal de los Zoophycos con composición externa al sustrato bioturbado. De la misma forma, tampoco se encaja en el modelo agrónomo de Bromley (1991), en que existen evidencias icnológicas del reprocesamiento del relleno fecal, posiblemente enriquecido, almacenado en partes específicas de las estructuras. Por sus características morfoetológicas, el modo de construcción de los ejemplares estudiados se aproxima, más bien, al más reciente modelo propuesto por Ekdale y Lewis (1991). Según este modelo, interpretado para los Zoophycos del Oligoceno de Nueva Zelanda, sus formas complejas son el resultado de una superposición protrusiva y tigmotáctica de bandas de alimentación del tipo Rhizocorallium (Ekdale y Lewis, 1991; Ekdale, 1992).

A través de un análisis morfológico se comprende las razones por las cuales los Zoophycos compuestos son estructuras fodinichnia complejas, muy eficientes cubriendo casi todo el volumen de sedimento disponible con propósitos nutricionales y un coste energético mínimo (Fig. 5).

\section{LA COMPETICIÓN INTRAESPECÍFICA PUEDE GENERAR HORMESIS: LA ASIMETRÍA DIRECCIONAL EN Zoophycos COMO MEDIDA PALEOECOLÓGICA DE INESTABILIDAD EN EL DESARROLLO}

Las facies de margocalizas con Zoophycos en offshore distal son muy abundantes en el Bajociense-Bathoniense del dominio del Tethys (p.e., Olivero, 1996). El icnogremio post-omisión de Zoophycos-Chondrites (Figura 4h), como expresión distal de la icnofacies de Glossifungites (cf. MacEachern y Burton, 2000), domina las facies de frente arrecifal/rampa homoclinal externa de las cuencas Lusitánica y del Algarve (vide Choffat, 1894; Rocha, 1976; Azerêdo, 1988; Duarte, 1991, 1997, 1998a, 1998b). En los niveles estudiados, los productores de Zoophycos aparecen en grandes densidades, ciertamente relacionado con ecotipos característicos de un ambiente intersticial disóxico, a veces microrreductor glauconítico, y un sustrato calciclástico (formado por clastos de composición carbonatada), firme y enriquecido en concentraciones orgánicas (bacterianas). La abundancia de Zoophycos, fácilmente observable a lo largo de todo el Toarciense-Calloviense del Algarve, parece estar asociada a ambientes de depósito caracterizados por una intermitencia entre períodos de baja o nula sedimentación después de eventos de flujos gravitacionales enriquecidos en nutrientes procedentes de niveles superficiales con mayor productividad orgánica (Olivero y Gaillard, 1996). Aunque las altas densidades de Zoophycos hayan sido interpretadas como oportunismo en condiciones de estrés ecológico (e.g., Miller y Johnson, 1981; Buatois et al., 1993; MacEachern y Burton, 2000), los ejemplos ahora estudiados no presentan características bióticas o ambientales de icnocenosis oportunistas, las cuales se manifies-

Figura 4. Aspectos morfoconstruccionales en los Zoophycos de la Praia da Mareta. a. Construcción helicoidal de galerías del tipo Rhizocorallium que genera el patrón de Zoophycos. b. Carácter protrusivo de las láminas. c. Lamelas con disposición centrípeta; el eje de enrollamiento está situado abajo de la base de la figura. d. Truncación erosiva del eje de enrollamiento. e. Zoophycos con crecimiento según el vector profundidad. f. Detalle de la estructura anterior. g.Lóbulo de prospección en sustrato desocupado. h. Colonización del sustrato firme por Chondrites a través de su superposición al patrón de bioturbación primaria de Zoophycos, explotando el sedimento entre las láminas. Escala $=5 \mathrm{~cm}$ (en a, b, e y f) y $2.5 \mathrm{~cm}(\mathrm{en}$ $\mathrm{c}, \mathrm{d}, \mathrm{g}$ y h).

Morpho-constructional features in Zoophycos from Praia da Mareta. a. Helicoidal construction of Rhizocorallium-type burrows that generates the Zoophycos pattern. $\boldsymbol{b}$. Protrusive character of lamina. $\boldsymbol{c}$. Spreite lamella with centripetal displacement; the coiling axis is situated below the base of the figure. $\boldsymbol{d}$. Erosive truncature of the coiling axis. $\boldsymbol{e}$. Zoophycos with growth according to the depth vector. $f$. Detail of the previous structure. $g$. Searching lobe within vacant substrate. $\boldsymbol{h}$. Occupation of the firmground by Chondrites overimposing the initial bioturbation pattern of Zoophycos. Scale bar $=$ $5 \mathrm{~cm}$ (in $a, b$, $e$ and $f$ ) and $2.5 \mathrm{~cm}$ (in $c, d$, $g$ and $h$ ). 


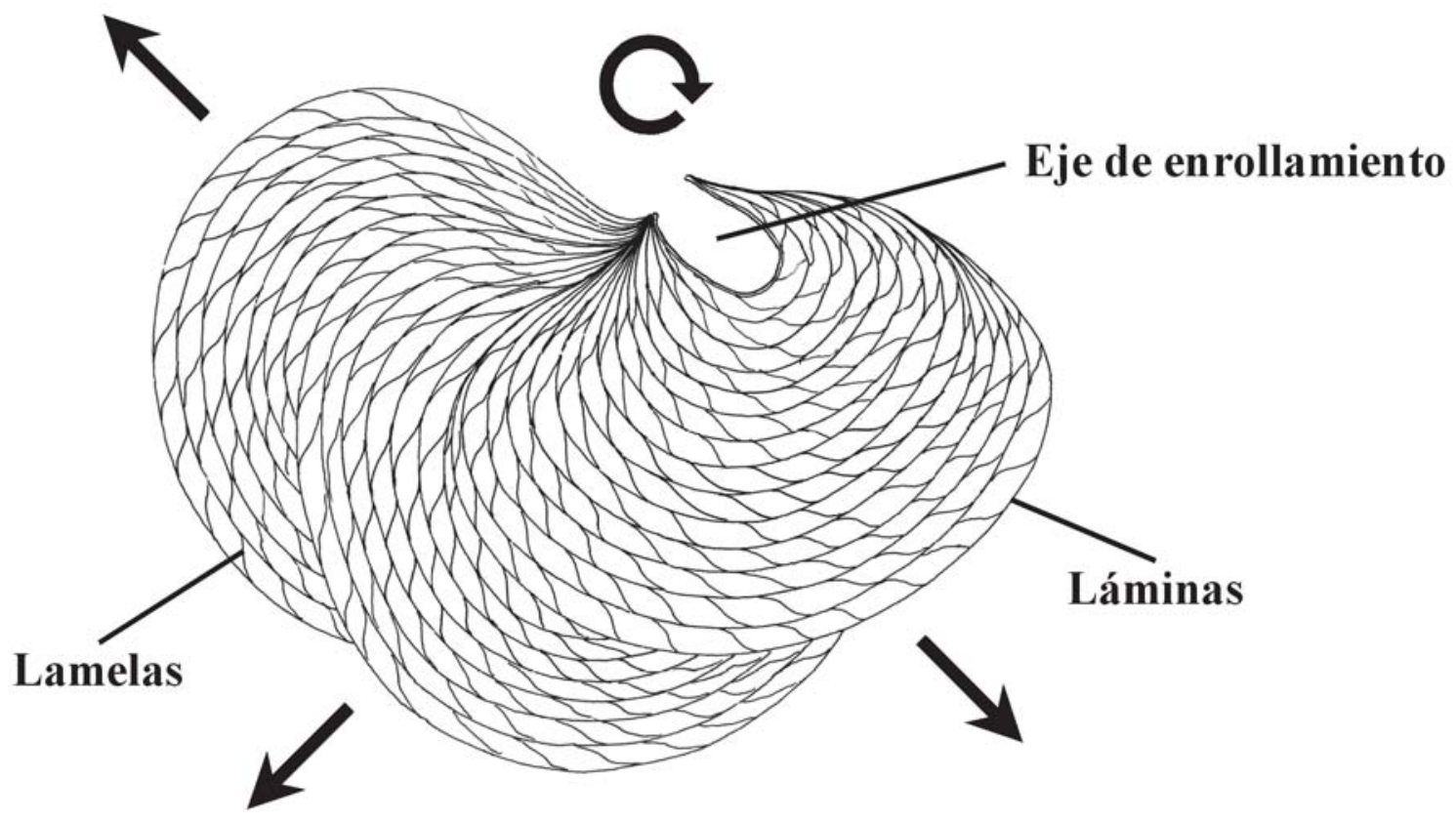

Figura 5. Modelo de construcción de los Zoophycos de la Praia da Mareta. Las flechas indican los vectores de crecimiento y la rotación se refiere al sentido de enrollamiento (dextrógiro).

Constructional model for the Zoophycos of Praia da Mareta. Arrows show the growth vectors and rotation refers to coiling direction (dextral).

tan por comportamientos sencillos, generalizados y nutricionalmente poco eficientes, mayoritariamente efímeros y poco diversificados, en respuesta a condiciones ambientales inestables en un largo espectro de tolerancia (Ekdale, 1985; Vossler y Pemberton, 1988). Se tratan más bien de icnocenosis post-omisión, pero con obliteración multigeneracional de las anteriores, ocupando estratégicamente, de forma prolongada y eficiente, la totalidad de un ecoespacio característico en el Jurásico, rico en nutrientes y estable desde el punto de vista de las condiciones de sedimentación (cf. Olivero y Gaillard, 1996).

Las icnocomunidades clímax de Zoophycos tienden a mostrar antisimetría ecofenotípica del enrollamiento. A lo largo de su desarrollo "ontogénico", el productor de Zoophycos realiza una prospección del sustrato en el sentido de la rotación de las agujas del reloj o contrario a éste, indefinidamente. De hecho, la proporción de 50:50 para enrollamientos dextrógiros y levógiros evita la superposición en icnofábricas densas. La ausencia de estímulos direccionales fuertes en el desarrollo del comportamiento es asumida como característica en estas estructuras (e.g., Miller y Johnson, 1981; Ekdale, 1992; Olivero y Gaillard, 1996), verificándose lo mismo en icnofábricas densas de Daedalus y Gyrolithes (formas de comportamiento helicoidales), asociadas a facies infralitorales, colonizando sustratos firmes del Ordovícico Medio y Mioceno Superior, respectivamente (Fig. 6).

El muestreo efectuado para el análisis de asimetrías en el enrollamiento de Zoophycos se ha realizado en el nivel estratigráfico NE2, que presenta un índice de biorturbación 5 (rellenando la totalidad del plano de estratificación) y un número elevado de estructuras en superposición. Por esta razón, el sentido de enrollamiento sólo pudo ser definido para las últimas formas de Zoophycos que han bioturbado este nivel (la última icnocenosis antes de los cambios ambientales que llevaron a la sedimentación de la capa suprayacente). Por este motivo, queda limitado al máximo el efecto negativo de la compactación diagenética en la metodología utilizada. En el gráfico de la Figura 6 se verifica un gran desfasamiento en las proporciones del sentido de enrollamiento para los Zoophycos presentes en NE2, comparando con los valores estadísticos obtenidos en los dos niveles con icnofábricas densas de Daedalus halli (Rouault, 1851) y Gyrolithes ichnospp. (icnoespecies de morfología helicoidal utilizadas para comparación con Zoophycos). El análisis de este desfasamiento resulta de la conjugación de los datos paleoicnológicos con la teoría de la biología evolutiva, relacionada con los estudios de dinámica no lineal.

Según Møller y Swaddle (1997), los fenotipos se desarrollan como resultado de la acción conjunta del sistema genético (determinista) y de los sistemas de desarrollo estocásticos (factores ambientales: físico-químicos y coevolutivos). La importancia de las condiciones iniciales del medio en la estructuración etológica efectuada a lo largo del desarrollo es característica de la dinámica biológica no lineal. Cuando existen desvíos de las condiciones ambientales respecto al óptimo ecológico, se ge- 


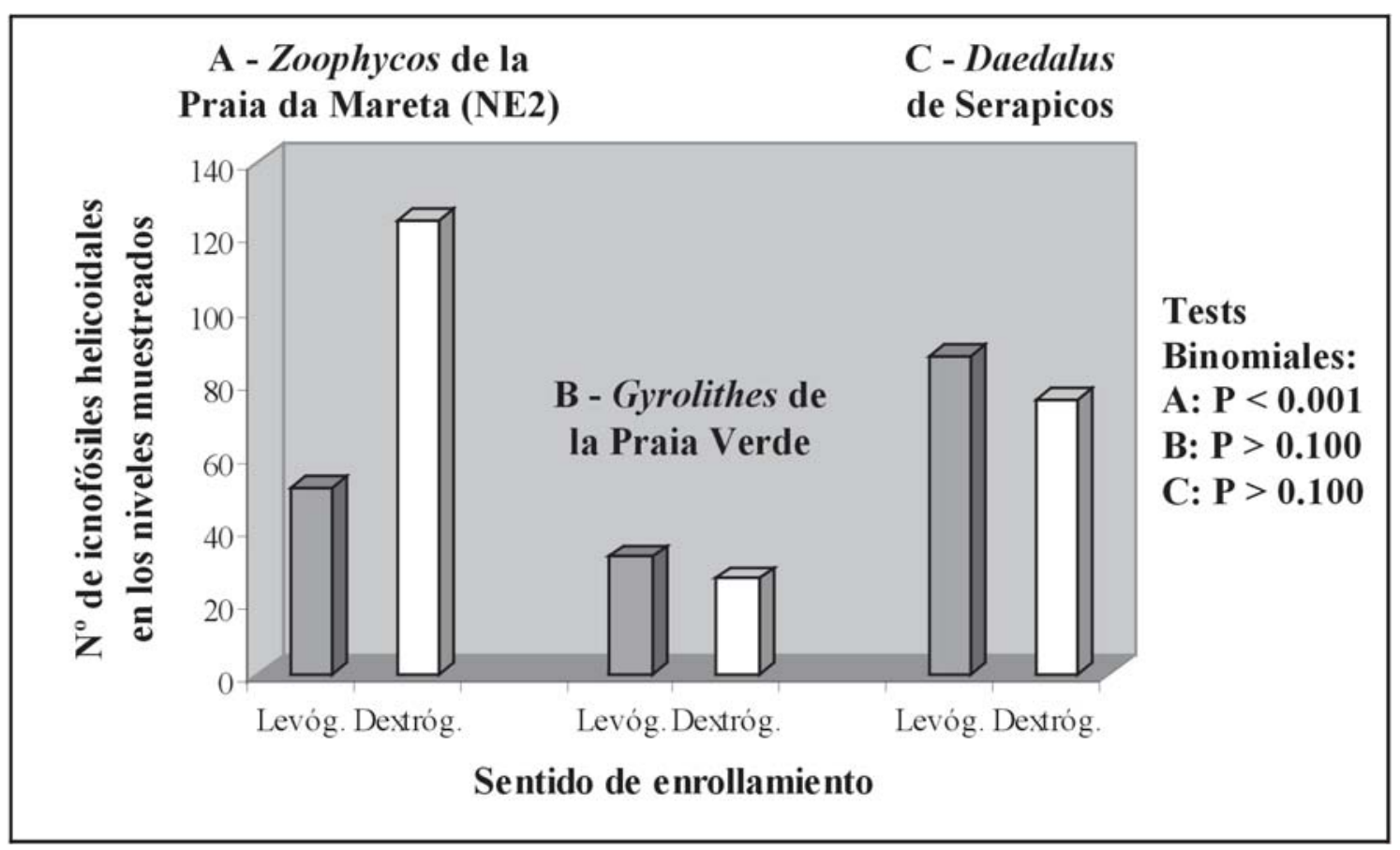

Figura 6. Gráfico de frecuencias del sentido de enrollamiento en asociaciones de icnofósiles helicoidales seleccionados (Zoophycos de NE2, Gyrolithes y Daedalus). En el análisis de probabilidad de la relación entre Zoophycos dextrógiros y levógiros se ha utilizado la distribución binomial.

Graph for coiling direction frequency in selected helicoidal ichnofossil assemblages (Zoophycos of NE2, Gyrolithes $y$ Daedalus). The probability analysis of the dextral-sinistral ratio used the binomial distribution test.

nera disipación energética, con la consecuente pérdida de plasticidad fisiológica y reajuste de los programas etológicos. El nivel de lateralización tiende a aumentar con la transición hacia el caos (Møller y Swaddle, 1997). De esta forma, y dado que en un organismo con simetría bilateral (la tienen todos los endobentónicos vágiles, por limitaciones biomecánicas en el morfoespacio), existen dos "cuencas de atracción comportamentales" adaptativas ("lado izquierdo" y "lado derecho"), la trayectoria del desarrollo del comportamiento en condiciones de estrés ambiental tiene tendencia a converger hacia una de estas opciones, promoviendo la instauración de asimetría direccional. Sin embargo, las observaciones realizadas (Fig. 6) contradicen estas ideas adaptacionistas, estando de acuerdo con las opiniones de Fürsich y Palmer (1984) encontradas en el estudio de las asimetrías ecofenotípicas en la deformación de la comisura en braquiópodos. De hecho, en el nivel estratigráfico NE2 de la Praia da Mareta, la aproximación a los límites de tolerancia de los productores de Zoophycos, interpretada por el incremento progresivo de la anoxia, depleción nutricional por superposición de sucesivas icnocenosis y/o restricción del ecoespacio por sobrepoblación (interdigitaciones, colisiones y superposiciones entre estructuras; Figs. 7a - 7d), habría llevado al desarrollo hormético de asimetría direccional en las últimas icnocenosis formadas en NE2 (relación entre Zoophycos dextrógiros y levógiros es de
$3: 1 ; \mathrm{P}<0.001 ; \mathrm{n}=175)$. En este caso, como en los ejemplos de Fürsich y Palmer (1984), que se alejan puntualmente de las líneas adaptativas darwinistas, no habrá habido cualquier significado genómico (evolutivo), ya que se ha verificado que las asimetrías direccionales no se han reproducido en las poblaciones en niveles estratigráficos sobreyacentes.

\section{EL DOMINIO ECOLÓGICO REFLEJADO EN LA ICNOFACIES DE Zoophycos: TENDENCIAS GLOBALES HACIA LA COLONIZACIÓN DE NICHOS MARINOS PROFUNDOS}

La figura 8 muestra diagramas cronológico-ambientales para Zoophycos, elaborados a partir de los datos conocidos para Portugal (diagrama de la izquierda), complementados por más de un centenar de referencias bibliográficas sobre Zoophycos de todo el mundo (diagrama de la derecha). La información ha sido agrupada en intervalos de $10 \mathrm{Ma}$., señalándose el total de referencias para cada ambiente marino (cf. Bottjer y Droser, 1994).

A partir de los datos conocidos referentes a Portugal, se constata que se produce la misma tendencia de radiación ecológica verificada en este comportamiento de ali- 

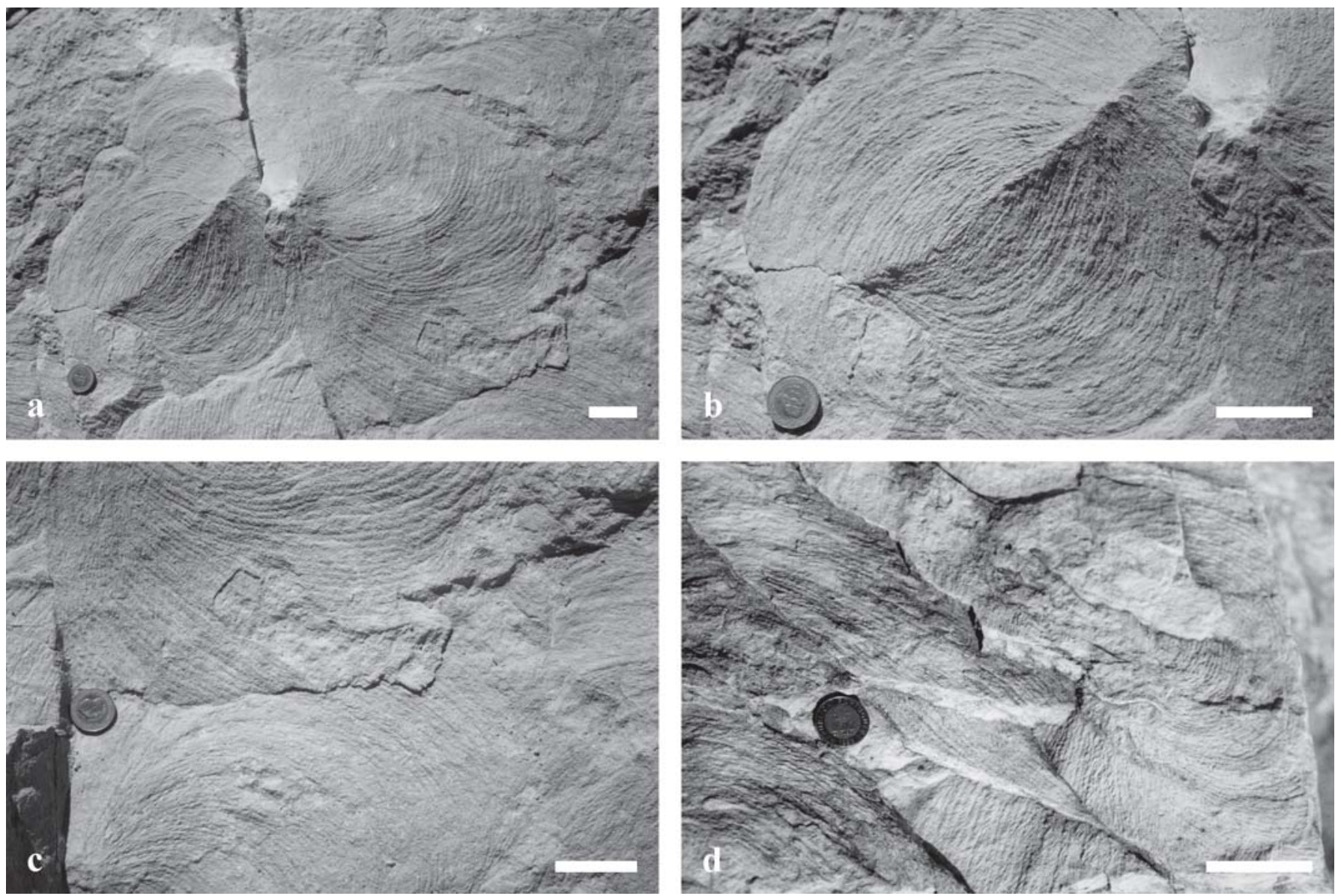

Figura 7. Interacciones ecológicas en las icnocenosis densas de Zoophycos. a. Colisión por crecimiento de Zoophycos. b. Detalle de las estructuras anteriores. c. Superposición de láminas de una misma icnocenosis. d. Cruce entre Zoophycos de dos icnocenosis. Escala $=5 \mathrm{~cm}$ (en todos los casos).

Ecological interactions in Zoophycos dense ichnocoenoses. $\boldsymbol{a}$. Collision by growth of Zoophycos. $\boldsymbol{b}$. Detail of the previous structures. c. Onlapping lamina within a single ichnocoenosis. d. Zoophycos cross-cuttings of two generations. Scale bar $=5 \mathrm{~cm}($ in all cases $)$.

mentación complejo para el resto del mundo, difiriendo estos, en algunos aspectos, de los datos obtenidos anteriormente por otros autores (p.e., Bottjer y Droser, 1994), los cuales se pasan a describir. Así, el productor de Zoophycos habría surgido en el inicio del Fanerozoico (en el Cámbrico Inferior existe una referencia de Bryant y Pickerill, 1990; en Portugal sólo aparece en el Arenig), en nichos infralitorales y marinos profundos (circalitoral en el sentido del paleofondo de la cuenca; nuevas y raras evidencias de este icnogénero en las turbiditas distales del Arenig superior de la Formación Xistos com Phyllodocites en Barrancos, Portugal). A partir del Arenig superior presenta un máximo ecológico en sustratos consistentes con situaciones de baja oxigenación intersticial, baja competición interespecífica en niveles de ocupación más o menos profundos y con influencia intermitente de materia orgánica transportada a partir de biótopos más superficiales y proximales. Durante el Paleozoico Superior, Zoophycos abunda globalmente en las facies lutíticas li- torales y de lagoon-deltaicas, aunque aparezca fortuitamente en ambientes batiales (cf. Droser y Bottjer, 1994). A partir del Toarciense, el productor de Zoophycos habría abandonado progresivamente los ambientes costeros a favor de profundidades mayores; la última referencia a la presencia de Zoophycos en ambiente de lagoon está datada en el Kimmeridgiense de la Cuenca Lusitánica (Fürsich, 1981). La desaparición de Zoophycos de la plataforma continental parece ocurrir, curiosamente, en los momentos próximos al gran evento de extinción del límite Cretácico-Terciario. Las razones para la erradicación de esta forma de comportamiento en la plataforma (de mayor productividad biológica) a favor de las zonas batialesabisales (de limitados recursos tróficos) pueden explicarse por la notable diversificación trófica en el Mesozoico, cuyo paradigma es el aumento de presión de los decápodos en todos los niveles de ocupación de los sustratos neríticos (ubiquidad del icnogremio de Thalassinoides) a lo largo del Jurásico-Cretácico, asociado al incremento de la pro- 


\section{Zoophycos en Portugal}

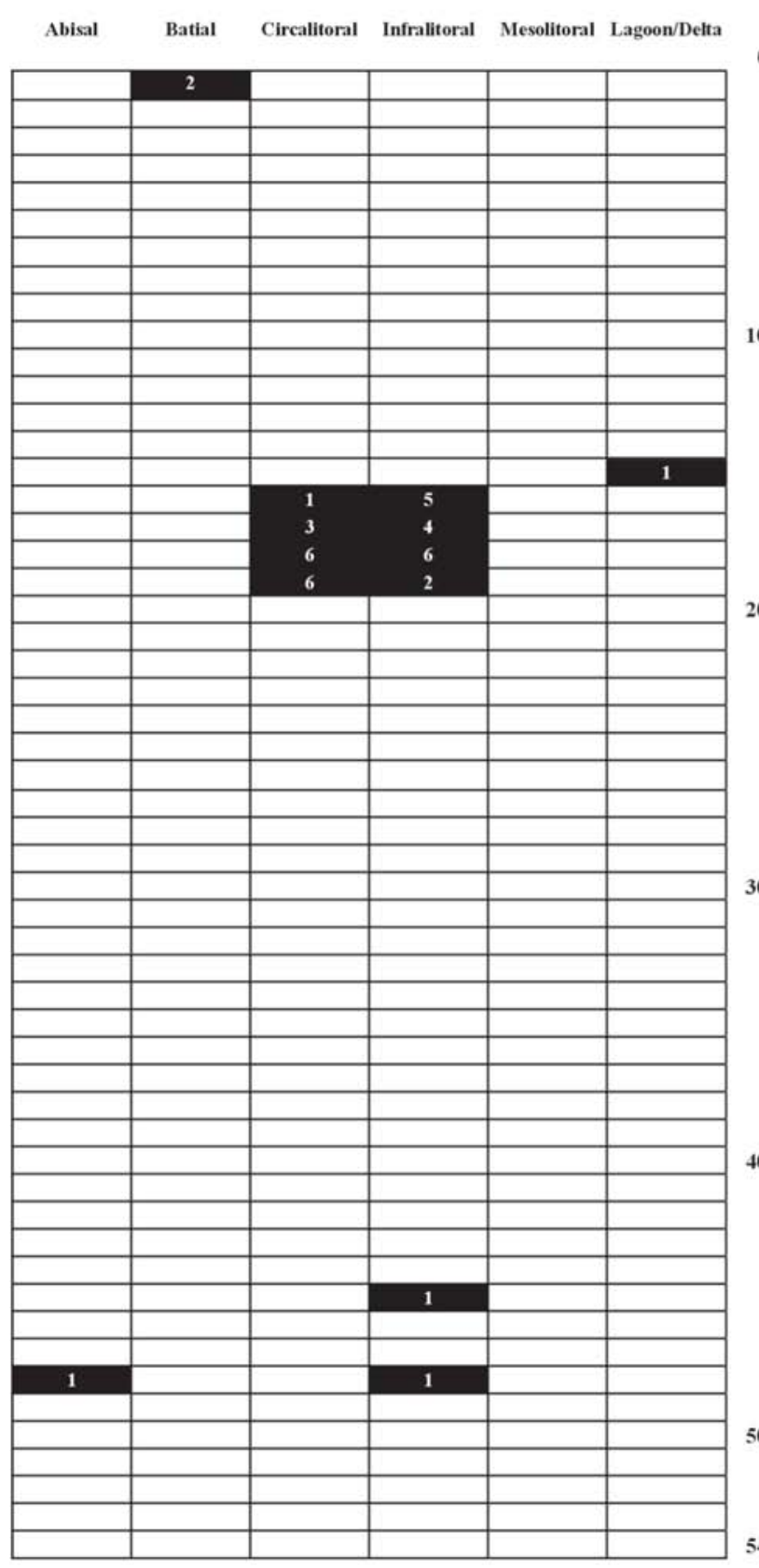

0

100

200

300

400

M.a.

\section{Zoophycos en el resto del mundo}

(1)

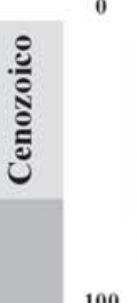

Abisal Batial Circalitoral Infralitoral Mesolitoral Lagoon/Delta
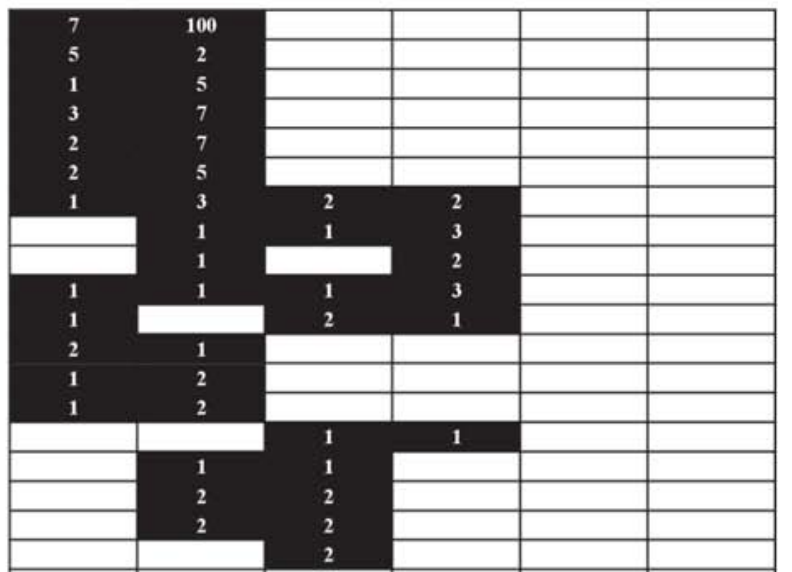

200

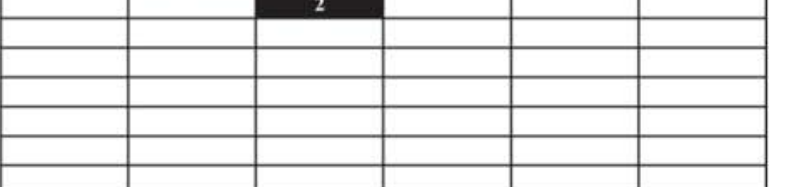

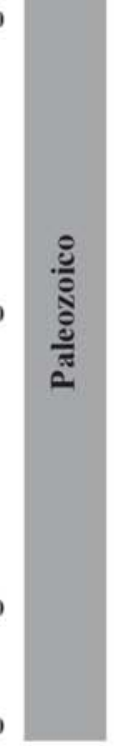

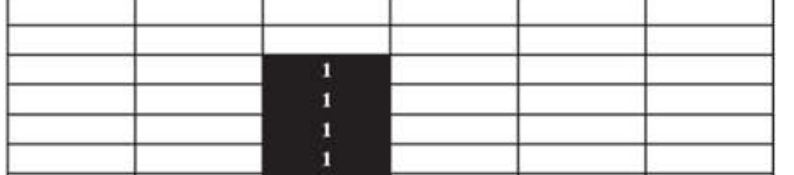

300

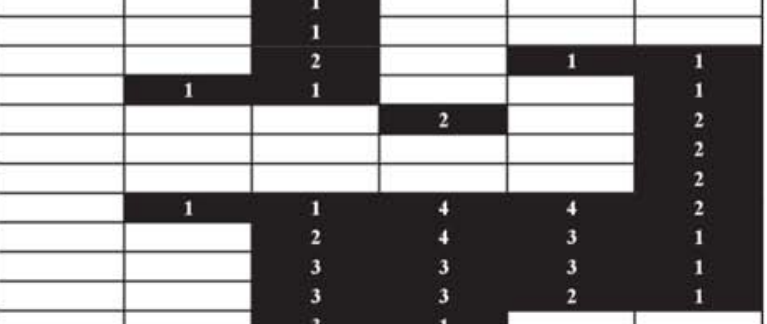

400

00

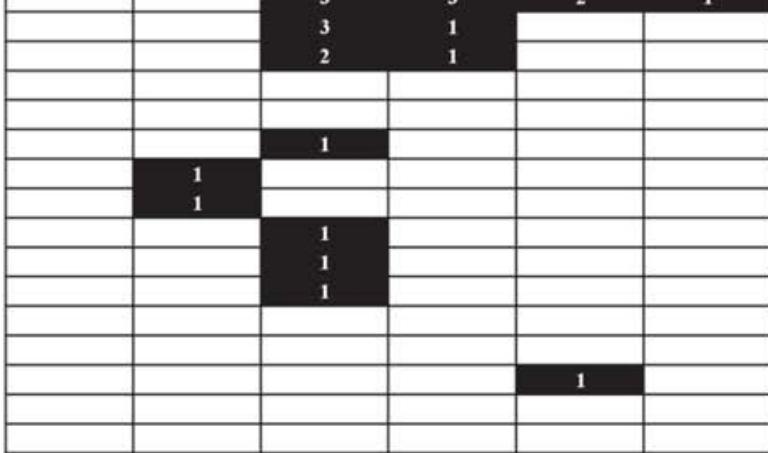

I.

Figura 8. Diagramas cronológico-ambientales para los Zoophycos hallados en el registro estratigráfico de Portugal (a la izquierda) y del resto del mundo (a la derecha). Los números indican la cantidad de publicaciones con referencias a Zoophycos analizadas para cada intervalo de tiempo y ambiente designados.

Time-environment diagrams for Zoophycos registered in Portugal (at left) and the rest of the world (at right). The numbers show the amount of publications with references to Zoophycos analysed for each chronological interval and environment designated.

ductividad orgánica pelágica en el medio oceánico (con la diversificación de la microfauna) y de la afluencia terrígena de fitodetritos en profundidad, sobre todo a partir del Cretácico Superior (Seilacher, 1974). El productor de Zoophycos habría sustituido la complejidad ocupacional del sustrato de plataforma por un ecoespacio de elevada simplicidad trófica y con fuertes analogías a los ecotipos conquistados en el Paleozoico. 


\section{AGRADECIMIENTOS}

Los autores agradecen la colaboración de Joana Ramos (Escola EB2, 3 de Castanheira do Ribatejo) y de Hugo Antunes en los trabajos de campo; Michael Romano (University of Sheffield) ha contribuido con datos inéditos referentes a Zoophycos de la Formación Quartzito Armoricano, encontrados en Portugal; Soledad Ureta Gil (Universidad Complutense de Madrid), bien como los dos revisores, Maria Helena Henriques (Universidade de Coimbra) y Federico Olóriz (Universidad de Granada), han contribuido con consejos para mejorar la comprensión del artículo; el colega Carlos Farinha ha dibujado el modelo interpretativo de la figura 5; una palabra de agradecimiento a Mário Cachão (Faculdade de Ciências da Universidade de Lisboa y Grupo Paleo) por las productivas discusiones y el incentivo a lo largo de este trabajo.

\section{BIBLIOGRAFÍA}

Azerêdo, A.C. 1988. Calcareous Debris-Flows as Evidence for a Distally Steepened Carbonate Ramp in West-Central Portugal. Comunicações dos Serviços Geológicos de Portugal, 74, 57-67.

Barbosa, B.P., Soares, A.F., Rocha, R.B., Manuppella, G. e Henriques, M.H. 1988. Carta Geológica de Portugal à escala 1:50000, Notícia Explicativa da Folha 19-A (Cantanhede). Serviços Geológicos de Portugal, Lisboa, 1-46.

Bottjer, D.J. and Droser, M.L. 1994. The history of Phanerozoic bioturbation. In: The Palaeobiology of Trace Fossils (Ed. S.K. Donovan). The Johns Hopkins University Press, Baltimore, 155-176.

Bromley, R.G. 1991. Zoophycos: stripe mine, refuse damp, cache or sewage farm? Lethaia, 24, 460-462.

Bryant, I.D. and Pickerill, R.K. 1990. Lower Cambrian trace fossils from the Buen Formation of central North Greenland: preliminary observations. In: Lower Cambrian trace fossils from Greenland (Ed. J.S. Peel). Grønlands Geologiske Undersøgelse Rapport, 147, 44-62.

Buatois, L.A., Martinioni, D.R. y Lirio, J.M. 1993. Trazas fósiles en una plataforma dominada por tormentas, Formación Rabot (Cretácico Sup., Punta Rabot, Isla James Ross, Antártida). Segundas Jornadas de Comunicaciones sobre Investigaciones Antárticas, Buenos Aires, 163-166.

Cachão, M., de Gibert, J.M., Mayoral, E., Muñiz, F. e da Silva, C.M. 2000. Paleoicnologia da Formação de Cacela (Miocenico Superior), Algarve, Portugal: dados preliminares. I Congresso Ibérico de Paleontología / XVI Jornadas de la Sociedad Española de Paleontología, Évora, 5-7.

Choffat, P. 1880. Étude stratigraphique et paléontologique des terrains jurassiques du Portugal. Le Lias et le Dogger au Nord du Tage. Mémoires de la Section des Travailles Géologiques du Portugal, 1-79.

Choffat, P. 1887. Recherches sur les terrains secondaires au sud du Sado. Comunicações da Commissão dos Trabalhos Geológicos de Portugal, 1 (2), 222-312.
Choffat, P. 1894. Notice stratigraphique sur les gisements de végétaux fossiles dans le Mésozoique du Portugal. In: Flore fossile du Portugal. Nouvelles contributions à la flore mésozoique (Ed. M. de Saporta). Comunicações da Direcção dos Trabalhos Geológicos de Portugal, Lisboa, 227-286.

Delgado, J.F.N. 1908. Système Silurique du Portugal. Étude de stratigraphie paléontologique. Memórias da Commissão do Serviço Geológico de Portugal, 1-245.

Delgado, J.F.N. 1910. Terrains paléozoïques du Portugal. Étude sur les fossiles des schistes à Néréites de San Domingos et des schistes à Néréites et à Graptolites de Barrancos. Memórias da Commissão do Serviço Geológico de Portugal, 1-68.

Duarte, L.V. 1991. Os margo-calcários do Toarciano na região de Rabaçal-Condeixa: caracterização sedimentológica e evolução sequencial. Memórias e Notícias, Publicações do Museu e Laboratório Mineralógico e Geológico da Universidade de Coimbra, 112 (A), 15-36.

Duarte, L.V. 1997. Facies analysis and sequential evolution of the Toarcian-Lower Aalenian series in the Lusitanian Basin (Portugal). Comunicações do Instituto Geológico e Mineiro, 83, 65-93.

Duarte, L.V. 1998a. O Liásico Superior de Peniche: Modelos de Sedimentação Autogenética Versus Alogenética. In: Livro Guia das Excursões, V Congresso Nacional de Geologia (Eds. J.T. Oliveira e R.P. Dias). Instituto Geológico e Mineiro, Lisboa, 21-25.

Duarte, L.V. 1998b. Hierarquização Sequencial em Sedimentos Margo-Calcários Alternantes. O Toarciano da Região do Rabaçal. In: Livro Guia das Excursões, V Congresso Nacional de Geologia (Eds. J.T. Oliveira e R.P. Dias). Instituto Geológico e Mineiro, Lisboa, 47-51.

Ekdale, A.A. 1985. Paleoecology of the marine endobenthos. Palaeogeography, Palaeoclimatology, Palaeoecology, 50, 63-81.

Ekdale, A.A. 1992. Muckraking and Mudslinging: The Joys of Deposit-Feeding. In: Trace Fossils (Eds. C.G. Maples and R.R. West). The Paleontological Society, University of Tennessee, Knoxville, Short Courses in Paleontology, 5, 145-171.

Ekdale, A.A. and Lewis, D.W. 1991. The New Zealand Zoophycos revisited: morphology, ethology, and paleoecology. Ichnos, 1, 183-194.

Fürsich, F.T. 1981. Invertebrate trace fossils from the Upper Jurassic of Portugal. Comunicações dos Serviços Geológicos de Portugal, 67, 153-168.

Fürsich, F.T. and Palmer, T.J. 1984. Commissural asymmetry in brachiopods. Lethaia, 17, 251-265.

Heer, O. 1881. Contributions à la flore fossile du Portugal. Mémoires de la Section des Travailles Géologiques du Portugal, 1-51.

Kotake, N. 1989. Paleoecology of the Zoophycos producers. Lethaia, 22, 327-341.

MacEachern, J.A. and Burton, J.A. 2000. Firmground Zoophycos in the Lower Cretaceous Viking Formation, Alberta: A Distal Expression of the Glossifungites Ichnofacies. Palaios, 15, 387-398. 
Manupella, G., Telles Antunes, M., Costa Almeida, C.A., Azerêdo, A.C., Barbosa, B., Cardoso, J.L., Crispim, J.A., Duarte, L.V., Henriques, M.H., Martins, L.T., Ramalho, M.M., Santos, V.F. e Terrinha, P. 2000. Carta Geológica de Portugal à escala 1:50000, Notícia Explicativa da Folha 27-A (Vila Nova de Ourém). Instituto Geológico e Mineiro, Lisboa, 1-156.

Miller, M.F. and Johnson, K.G. 1981. Spirophyton in alluvialtidal facies of the catskill deltaic complex: possible biological control of ichnofossil distribution. Journal of Paleontology, 55, 1016-1027.

Miller, M.F. and Smail, S.E. 1997. A Semiquantitative Field Method for Evaluating Bioturbation on Bedding Planes. Palaios, 12, 391-396.

Møller, A.P. and Swaddle, J.P. 1997. Asymmetry, Developmental Stability, and Evolution. Oxford University Press, 1-291.

Olivero, D. 1996. Zoophycos distribution and sequence stratigraphy. Examples from the Jurassic and Cretaceous deposits of southeastern France. Palaeogeography, Palaeoclimatology, Palaeoecology, 123, 273-287.

Olivero, D. and Gaillard, C. 1996. Paleoecology of Jurassic Zoophycos from south-eastern France. Ichnos, 4, 249-260.

Piçarra, J.M. 2000. Estudo estratigráfico do Sector de EstremozBarrancos, Zona de Ossa Morena, Portugal. Vol. II - Bioestratigrafia do intervalo Ordovícico-Devónico Inferior. Tese de Doutoramento (inédito), Universidade de Évora, 1-173.
Ribeiro, A. 1974. Contribution à l'étude tectonique de Trásos-Montes oriental. Memórias dos Serviços Geológicos de Portugal (Nova Série), 24, 1-168.

Rocha, R.B. 1971. Contribuição para o estudo geológico da região do Cabo de S. Vicente-Alvor. Boletim da Sociedade Geológica de Portugal, 17, 139-168.

Rocha, R.B. 1976. Estudo estratigráfico e paleontológico do Jurássico do Algarve ocidental. Ciências da Terra (UNL), 2, 1-178.

Ruget-Perrot, C. 1961. Études stratigraphiques sur le Dogger et le Malm inférieur du Portugal au Nord du Tage. Memórias dos Serviços Geológicos de Portugal (Nova Série), 7, 1-197.

Seilacher, A. 1974. Flysch trace fossils: Evolution of behavioural diversity in the deep-sea. Neues Jahrbuch für Geologie und Paläontologie, Monatshefte, 4, 233-245.

Teixeira, C. 1978. O mundo prodigioso dos fósseis. IV Curso de Extensão Universitária de Ciências Geológicas da Faculdade de Ciências da Universidade de Lisboa, 99-194.

Vossler, S.M. and Pemberton, S.G. 1988. Skolithos in the Upper Cretaceous Cardium Formation: an ichnofossil example of opportunistic ecology. Lethaia, 21, 351-362.

Wetzel, A. and Werner, F. 1981. Morphology and ecological significance of Zoophycos in deep-sea sediments of NW Africa. Palaeogeography, Palaeoclimatology, Palaeoecology, 32, 185-212.

Manuscrito recibido: 19 de diciembre, 2002 Manuscrito aceptado: 31 de julio, 2003 